\title{
New technologies for low-grade glioma
}

\section{surgery}

\author{
Nicolae-Ștefan Bogaciu ${ }^{1,3}$, Daniel Teleanu ${ }^{1,2,3}$, A.V. Ciurea ${ }^{2,3}$ \\ ${ }^{1}$ University Emergency Hospital, Bucharest, ROMANIA \\ 2 Sanador Clinical Centre Hospital, Bucharest, ROMANIA \\ 3 "Carol Davila" University of Medicine and Pharmacy, Bucharest, \\ ROMANIA
}

\begin{abstract}
Brain surgery has come far from the primitive methods used thousands of years ago. In the last hundred years alone, we have come across countless breakthroughs like the invention of bipolar coagulation, the surgical microscope, microsurgical techniques and a large variety of surgical instruments. The latest years have brought us neuro-navigation, intra-operative imaging techniques and brain activity monitoring. Low-grade glioma surgery, in particular, has taken advantage a lot from all these new methods, bringing great benefits for the patients: the safe extent of resection has grown progressively and tumours located in eloquent areas that were thought until recently to be inoperable, have started to be removed in safe conditions. The purpose of this paper is to present these new technologies as a recap for neurosurgical professionals.
\end{abstract}

\section{INTRODUCTION}

Endovascular Brain surgery is one of the oldest procedures to ever be performed on a human being. Beginning as trephining, it has known a long period of slow development, only to become one of the top-edge sciences of modern time. It developed from somewhat barbaric procedures with little if any supporting scientific knowledge, to lifesaving procedures performed on a macroscopic scale, to microscopic interventions with progressively smaller "collateral damage" and in the future it might turn into something we would call "molecular surgery". The purpose of this paper is to highlight the technologies we nowadays use in low-grade glioma surgery, presenting their pearls and pitfalls, benefits and uses.

\section{MATERIALS}

Computer assisted neuro-navigation. The purpose of this tool in brain surgery is to integrate imaging studies obtained preoperatively with intra-op anatomy in order to localize more accurately the lesions subdue to surgery (PICTURE 1). A neuro-navigation system has the following main parts: a computer-station with built-in software, a a receiver and trackers/pointers (PICTURE 2 ).

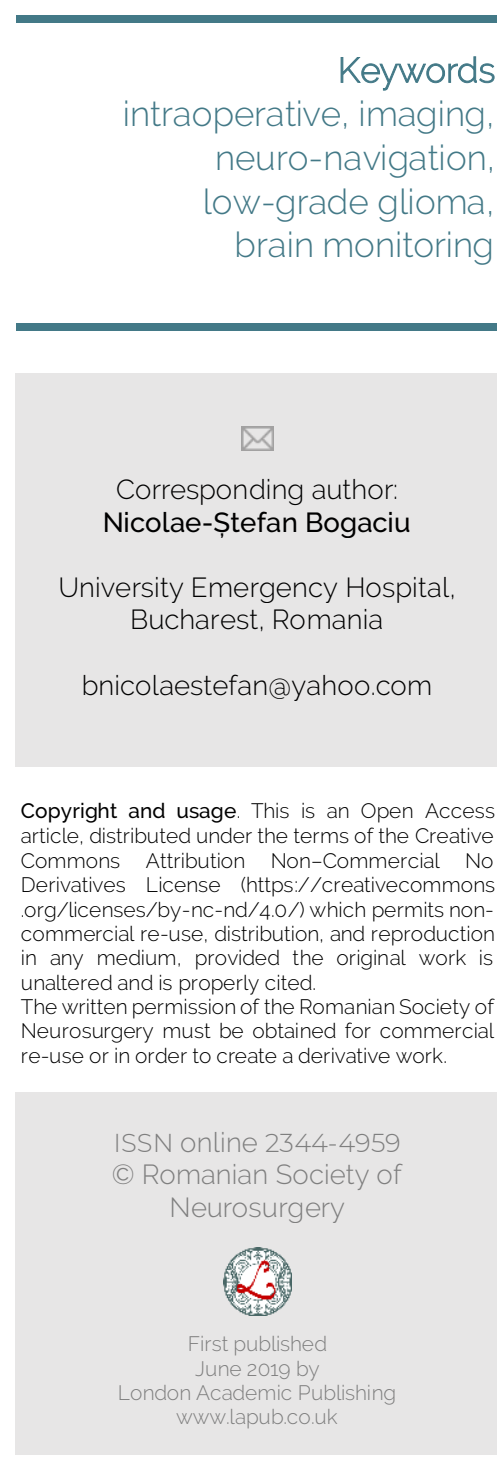




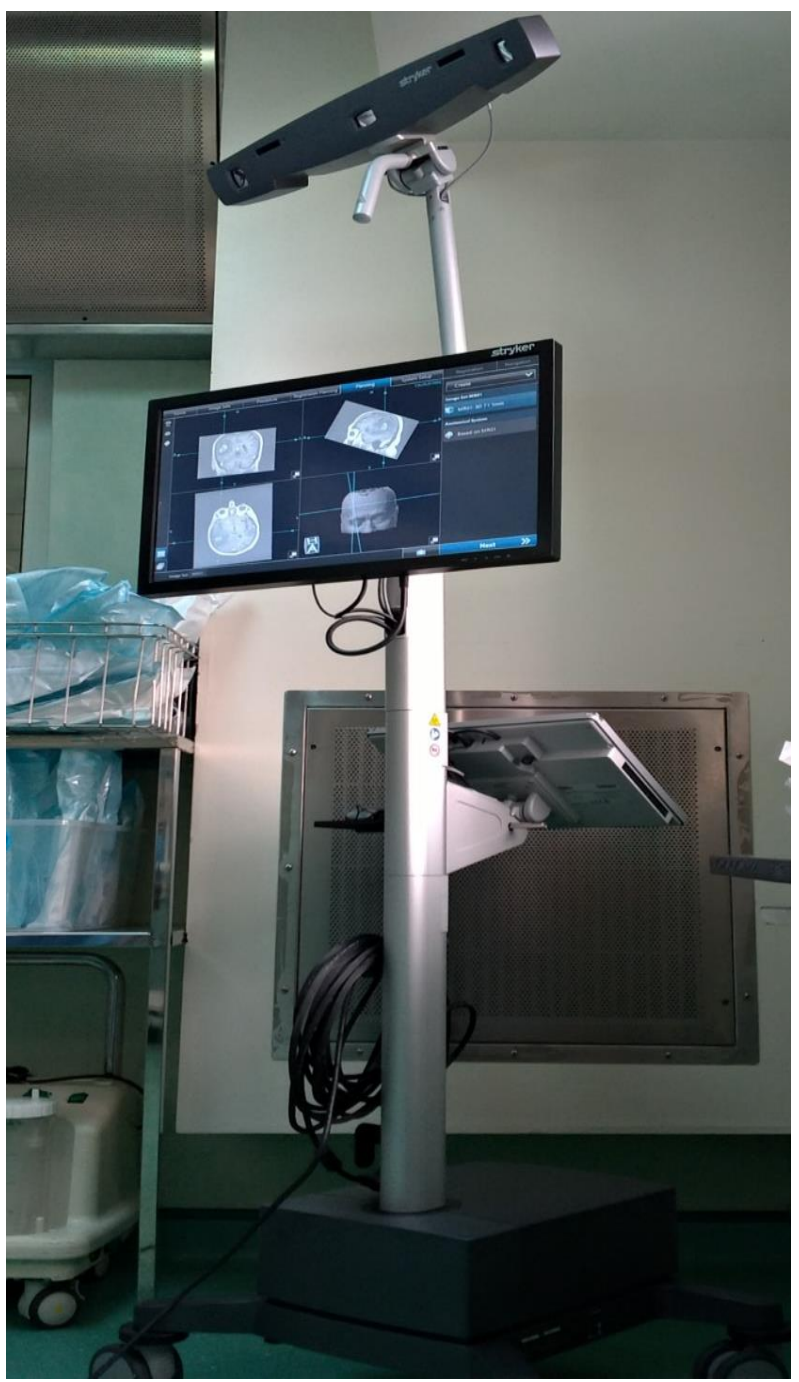

PICTURE 1

\section{PICTURE 2}

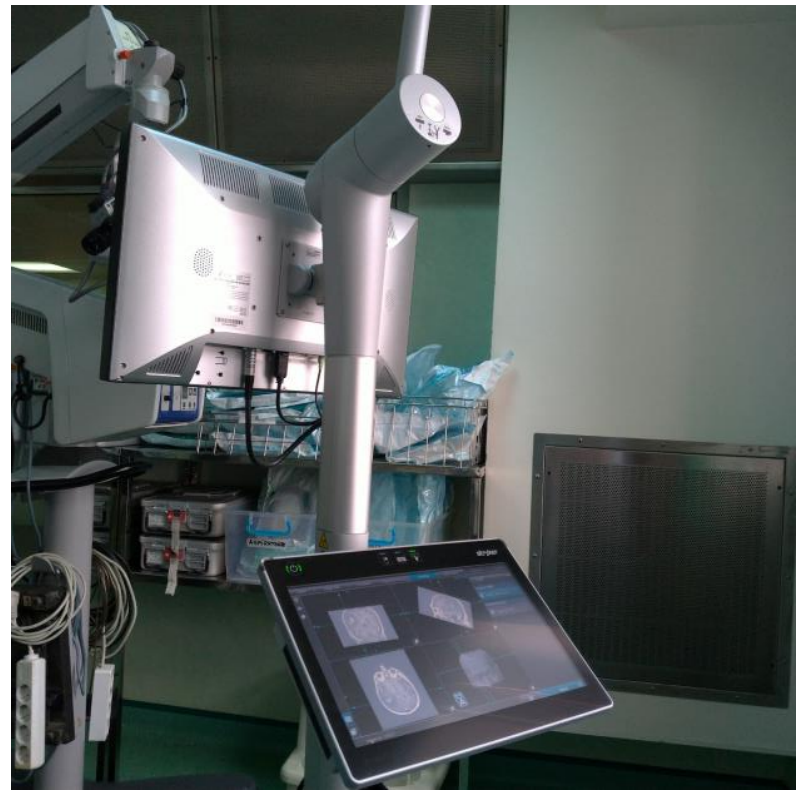

The initial step is to upload the preoperative images in the computer. Patient is positioned and a patienttracker is placed in the proximity of the patient's head. Landmark registration is performed and checked for accuracy and surgery can commence. Intra-operatively, the surgeon may navigate with different tools: pointers, microscope, standard neuro-navigated instruments (aspirator, ultrasonic aspirator, retractors etc.). Some systems allow injection of images directly into the microscope for compatible devices (PICTURE 3).

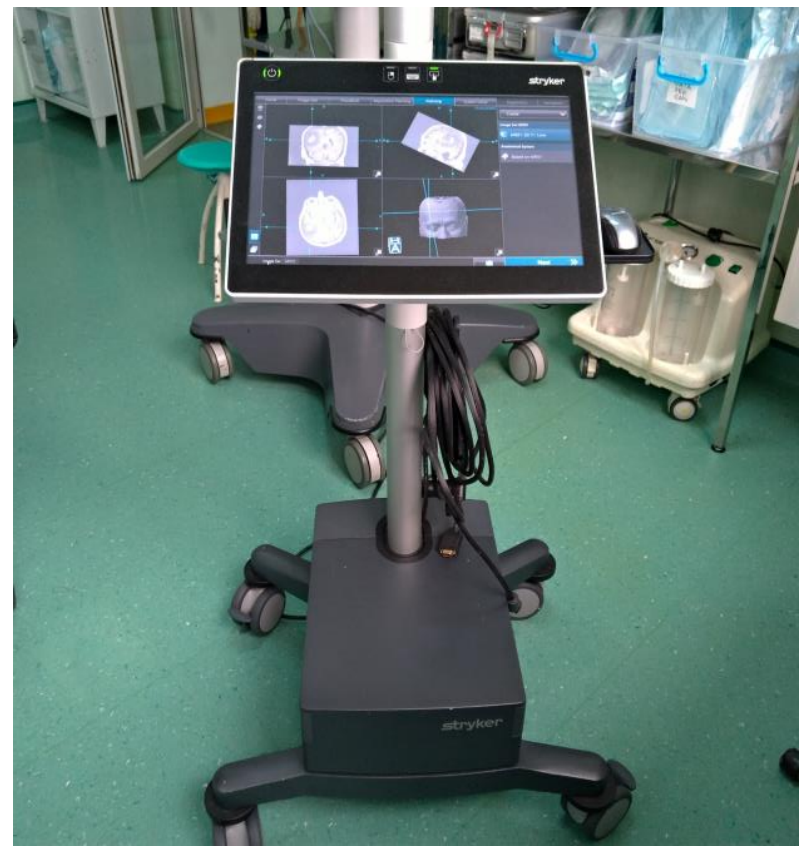

PICTURE 3

Neuro-navigation is not only useful for accurately localizing a lesion, but also for localizing vasculature and other normal anatomical structures that need to be avoided in glioma surgery. Image series used for neuro-navigation can be fused for better describing the anatomy and functionality of different brain areas: tractography, PET, MRI, CT, contrast-enhanced imagery etc. The advantages of using neuronavigation in LGG (low-grade glioma) surgery consist of, but are not limited to: smaller scalp incisions, cranial flaps, dural incisions and corticotomies, safer dissection and tumour excision, reduced surgery time.

All these imply fewer post-operative complications from surgery, faster discharge, lower costs for patient care. The main disadvantage of neuronavigation systems is that the position of the patient 
when obtaining images and the position of the patient during surgery are almost never the same so the brain will have a shift during surgery compared to imaging. Another thing is that after beginning the excision of tumoral tissue, the landscape changes, but the images stay the same. Some solutions were developed for these problems. Other imaging studies may be performed intraoperatively to complete the preoperative study and to increase the accuracy of the neuro-navigation system. Intraoperative MRI is a known tool which performs the task well, but it is highly inconvenient. Its more readily available counterpart is intraoperative ultrasound. Tips for successfully removing tumours using neuro-navigation include: removing tumour tissue closer to eloquent areas first, when accuracy is better, performing en-bloc excisions as much as possible, avoiding cysts especially when they are large, until good accuracy is no longer an issue, "rebuilding" volume by placing cottonoids in resulting cavities. In spite of all issues, a surgeon will always find this tool useful and he will always have in mind the fact that he can never rely on solely this in performing his tasks.1,2,3,4 (PICTURE 4).

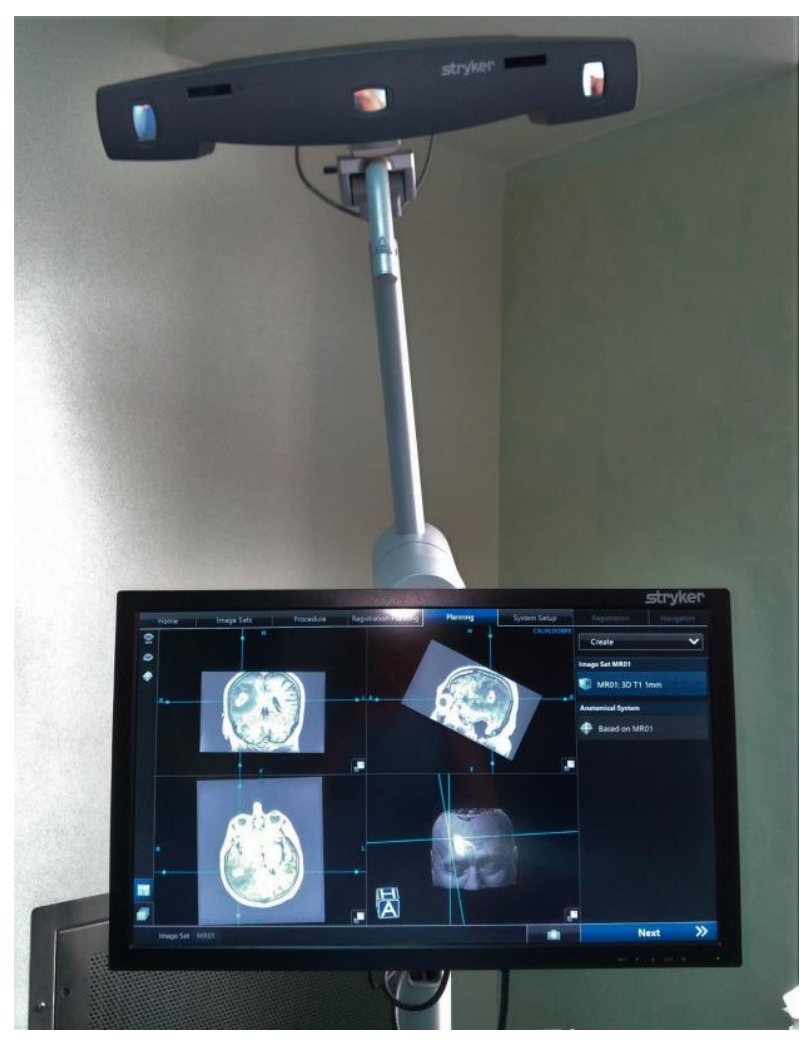

Intra-operative ultrasound. Ultrasonography (US) is a great mean of obtaining fast, cheap real-time images during brain surgery. Images are usually obtained by using a probe of $4-8 \mathrm{MHz}$ which is sufficient for acquiring images in a 2-6 cm distance from it. There are a few ways to use this tool: regular gray-scale brightness mode (B-mode), Contrast Enhanced Ultrasound (CEUS), elastosonography. US is of aid in all stages of brain tumour surgery, from the opening of the dura up to the resection of peripheral extensions of the tumour. As opposed to cortical lesions, subcortical lesions are more difficult to identify, and the site of the corticotomy may be tricky to choose. By using US, the surgeon can identify the subcortical tumour and choose an entry site as found suitable. During resection, the surgeon may use US to identify the depth and extent of the tumour and adapt the aggressivity of his resection accordingly. In the final stages of the surgery, one may use US for identifying residual tumoral tissue in the periphery of the mass. Not only US may be used for identifying the tumour, but also to characterize it. It can deliver information about the consistency of the tumour. For example, the surgeon may use this information to adapt the power of an ultrasonic aspirator, or to differentiate between low-grade glioma (LGG) and high-grade glioma (HGG): LGG are firmer than HGG (as assessed by elastosonography). CEUS brings even more information about the vascularization of the tumour, by real-time angiosonography so that the surgeon may adapt his resection strategy and have even more information about the possible histological diagnosis (LGG are poorly vascularized as opposed to HGG). A common use for intraoperative US in brain surgery is enhancing accuracy of neuro-navigation systems by updating the pre-operative acquired images used for registration and navigation, with real-time images provided by intra-operative US. By doing so, the effects of brain shift and selling may be partly counteracted.1,4-8

Intraoperative CT. Although it is less preferred by neurosurgeons compared to other imagistic tools, intraoperative CT-scans may be found useful in glioma surgery. As the other intraoperative imaging studies (US, MRI), it allows a greater extent of surgical resection by providing real-time intra-operative information regarding tumour mass, as well as updating pre-operative neuro-navigation planning. We may consider some advantages regarding the cost (relatively low compared to intraoperative MRI), speed of the procedure and general benefits such as improving accuracy and extent of the resection.9,10 
Intraoperative MRI (ioMRI). Intraoperative magnetic resonance imaging is the preferred intraoperative imaging method for low-grade glioma surgery. It is also the most laborious, the most expensive, but more importantly the most accurate. IoMRI comes in two shapes: low field ( $\leq 0,5$ Tesla) and high field $(\geq 1,5$ Tesla). Regardless of the power of the magnet, ioMRI aids the surgeon in extending tumour resection in both enhancing and non-enhancing lesions, with greater benefit in the latter. The use of intraoperative MRI associates with higher extent of resection which implies longer overall survival and free of symptoms survival in patients with gliomas. Setting up an operating room with an MRI device can get pretty challenging. Although there are tools that can be used within the area closest to the device (an area limited by the 5-gauss border, which is a line beyond which ferromagnetic instruments can be used), most instruments used for microsurgical resection, including microscope and ultrasonic aspirator, have to be used further away from the magnet. Residual tumour is observed with high fidelity on images acquired from intraoperative MRI and can be readily excised if feasible. The whole range of sequences can be studied: functional, connective or pathological areas can be identified. Newly acquired images are integrated into navigation systems making surgeon orientation precise.3,11-13

Intraoperative brain mapping and awake craniotomy. Although neuroimaging techniques have a great contribution to identifying functional cerebral structures and their relationship to tumor masses, intraoperative brain mapping is the gold standard for establishing tumour surgical strategy for each individual case, especially in the case of low grade glioma (LGG) where it is commonly accepted that the extent of resection (EOR) is linked to increase in survival. Intraoperative electric stimulation is performed by using a bipolar probe with an electric intensity starting from $2 \mathrm{~mA}$, with increments of $1 \mathrm{~mA}$ up to $6 \mathrm{~mA}$ in patients operated with local anaesthesia and up to $16 \mathrm{~mA}$ in patients operated with general anaesthesia. During the procedure, when a certain area of the brain is stimulated, it will produce a neurological disturbance which returns to normal after stimulation is stopped. Thereby the interested function is noted and the neurosurgeon can make a surgical decision of stopping the resection. Areas which when stimulated do not generate a disturbance are considered safe for continuing resection. LGG are slow-growing and rarely present with major neurological deficits, so the benefit of intraoperative brain mapping is large in LGG surgery because you need to preserve functionality for the patient. Even more, tumour mass resection is considered to be linked to postoperative seizure control so the possibility of safely increasing the EOR with this procedure increases the chance of good seizure control. Intraoperative brain mapping even offers the opportunity for safe tumour resection in eloquent areas otherwise considered inoperable. An important factor that contributes to this fact is brain plasticity. Brain plasticity also offers the opportunity for re-intervention in LGG of eloquent areas where initial total resection could not be performed, but timing of such a re-intervention must be carefully planned in order not to be too late and face anaplastic transformation.10,14-16

\section{CONCLUSIONS}

Low-grade glioma is a challenging pathology in the field of neurosurgery due to its infiltrative nature, especially when concerning eloquent areas of the brain. Safe resection of such lesions can be performed using a variety of instruments and procedures, but better results can be obtained by combining them. The goal is obtaining an increased extent of resection which is beneficial for the patient, improving survival and quality of life.

\section{REFERENCES}

1. Khoshnevisan A, Allahabadi NS. Neuronavigation: principles, clinical applications and potential pitfalls. Iran J Psychiatry. 2012;7(2):97-103. https://www.ncbi.nlm.nih.gov /pubmed/22952553.

2. Ricciardi L, Della Pepa GM, Izzo A, et al. Use of Neuronavigation System for Superficial Vein Identification: Safe and Quick Method to Avoid Intraoperative Bleeding and Vein Closure: Technical Note. World Neurosurg. 2018;117:92-96. doi:10.1016/j.wneu.2018.06.018

3. Lu C-Y, Chen X-L, Chen X-L, Fang X-J, Zhao Y-L. Clinical application of $3.0 \mathrm{~T}$ intraoperative magnetic resonance combined with multimodal neuronavigation in resection of cerebral eloquent area glioma. Medicine (Baltimore). 2018;97(34):e11702. doi:10.1097/MD.0000000000011702

4. Unsgaard G, Ommedal S, Muller T, Gronningsaeter A, Nagelhus Hernes TA. Neuronavigation by intraoperative three-dimensional ultrasound: initial experience during brain tumor resection. Neurosurgery. 2002;50(4):804-12; discussion 812. 
5. Pino MA, Imperato A, Musca I, et al. New Hope in Brain Glioma Surgery: The Role of Intraoperative Ultrasound. A Review. Brain Sci. 2018;8(11). doi:10.3390/brainsci8110202

6. Del Bene M, Perin A, Casali C, et al. Advanced Ultrasound Imaging in Glioma Surgery: Beyond Gray-Scale B-mode. Front Oncol. 2018;8:576. doi:10.3389/fonc.2018.00576

7. Prada F, Del Bene M, Rampini A, et al. Intraoperative Strain Elastosonography in Brain Tumor Surgery. Oper Neurosurg (Hagerstown, Md). November 2018. doi:10.1093/ons /opy323

8. Altieri R, Melcarne A, Di Perna G, et al. Intra-Operative Ultrasound: Tips and Tricks for Making the Most in Neurosurgery. Surg Technol Int. 2018;33:353-360.

9. Coburger J, Nabavi A, Konig R, Wirtz CR, Pala A. Contemporary use of intraoperative imaging in glioma surgery: A survey among EANS members. Clin Neurol Neurosurg. 2017;163:133-141. doi:10.1016/j.clineuro.2017. 10.033

10. Barbagallo GM V, Morrone A, Certo F. Intraoperative Computed Tomography and Awake Craniotomy: A Useful and Safe Combination in Brain Surgery. World Neurosurg. 2018;119:e159-e166. doi:10.1016/j.wneu.2018.07.078

11. Arya N, Stark AM, Lutz D, Mehdorn HM. Surgical Navigation with Intraoperative Imaging: Special Operating Room Concepts. In: Quiñones-Hinojosa A, ed. Schmidek \& Sweet Operative Neurosurgical Techniques: Indications, Methods, and Results. 6th ed. Philadelphia PA: Elsevier/Saunders; 2012:14-20.

12. Whiting BB, Lee BS, Mahadev $\mathrm{V}$, et al. Combined use of minimal access craniotomy, intraoperative magnetic resonance imaging, and awake functional mapping for the resection of gliomas in 61 patients. J Neurosurg. January 2019:1-9. doi:10.3171/2018.9.JNS181802

13. Mohammadi AM, Sullivan TB, Barnett GH, et al. Use of highfield intraoperative magnetic resonance imaging to enhance the extent of resection of enhancing and nonenhancing gliomas. Neurosurgery. 2014;74(4):339-350. doi:10.1227/NEU.0000000000000278

14. Gravesteijn BY, Keizer ME, Vincent AJPE, Schouten JW, Stolker RJ, Klimek M. Awake craniotomy versus craniotomy under general anesthesia for the surgical treatment of insular glioma: choices and outcomes. Neurol Res. 2018;40(2):87-96. doi:10.1080/01616412.2017.1402147

15. Frati A, Pesce A, Palmieri M, et al. Hypnosis-Aided Awake Surgery for the Management of Intrinsic Brain Tumors versus Standard Awake-Asleep-Awake Protocol: A Preliminary, Promising Experience. World Neurosurg. 2019;121:e882-e891. doi:10.1016/j.wneu.2018.10.004

16. Duffau H. Cortical and Subcortical Brain Mapping. In: Quiñones-Hinojosa A, ed. Schmidek \& Sweet Operative Neurosurgical Techniques: Indications, Methods, and Results. 6th ed. Philadelphia PA: Elsevier/Saunders; 2012:80-92. 